\title{
Study of the combined effects of data assimilation and grid nesting in ocean models - application to the Gulf of Lions
}

\author{
L. Vandenbulcke ${ }^{1}$, A. Barth ${ }^{2}$, M. Rixen ${ }^{3}$, A. Alvera-Azcarate ${ }^{2}$, Z. Ben Bouallegue ${ }^{1}$, and J. M. Beckers ${ }^{1}$ \\ ${ }^{1}$ GeoHydrodynamics and Environment Research, Université de Liège, Belgium \\ ${ }^{2}$ Ocean Circulation Group, University of South Florida, College of Marine Science. Florida 33701, USA \\ ${ }^{3}$ NATO/SACLANT Undersea Research Centre, La Spezia, Italy
}

Received: 27 March 2006 - Published in Ocean Sci. Discuss.: 18 May 2006

Revised: 28 August 2006 - Accepted: 28 October 2006 - Published: 31 October 2006

\begin{abstract}
Modern operational ocean forecasting systems routinely use data assimilation techniques in order to take observations into account in the hydrodynamic model. Moreover, as end users require higher and higher resolution predictions, especially in coastal zones, it is now common to run nested models, where the coastal model gets its opensea boundary conditions from a low-resolution global model. This configuration is used in the "Mediterranean Forecasting System: Towards environmental predictions" (MFSTEP) project. A global model covering the whole Mediterranean Sea is run weekly, performing 1 week of hindcast and a 10day forecast. Regional models, using different codes and covering different areas, then use this forecast to implement boundary conditions. Local models in turn use the regional model forecasts for their own boundary conditions. This nested system has proven to be a viable and efficient system to achieve high-resolution weekly forecasts. However, when observations are available in some coastal zone, it remains unclear whether it is better to assimilate them in the global or local model. We perform twin experiments and assimilate observations in the global or in the local model, or in both of them together. We show that, when interested in the local models forecast and provided the global model fields are approximately correct, the best results are obtained when assimilating observations in the local model.
\end{abstract}

\section{Introduction}

For various reasons, such as insufficiently known initial conditions, model parameters and atmospheric forcings, numerical ocean models progressively drift away from the true state of the ocean state. With the availability of numerous, often real-time or almost real-time observations, data assimilation

Correspondence to: L. Vandenbulcke

(luc.vandenbulcke@ulg.ac.be) techniques have proven to be an essential component of operational forecasting systems, as they allow to find a compromise (in some optimal way) between model forecasts and observations. Moreover, end users often ask for high resolution forecasts in coastal zones. A common technique to achieve this is to use nested grids, achieving high resolution results only in a limited region and thus avoiding the computational cost of high resolution in the rest of the basin. After choosing a specific data assimilation scheme, and a specific implementation of the nesting procedure, the combination of both those techniques can be realized in different ways. In particular, the available observations could be assimilated in the coarse, global model, or in the high resolution, local model. When assimilated in the global model, the information they bring would be transferred to the local model through initial and boundary conditions; when they are assimilated in the local model, they immediately have an impact on this models output; but the coarse resolution model, which is not corrected, might then feed the local model with inappropriate boundary conditions. The current study presents series of twin experiments in order to address those questions, under the hypothesis that we are interested mainly in the output of the high resolution model.

In the following section, we will describe our study area, the GHER hydrodynamic model, the grid nesting procedure, and the data assimilation scheme. We will also briefly show the results of the (unforced) nested model and compare with observations from the literature. The twin experiments are described in Sect. 3. In Sect. 4, we expose the different possible combinations of grid nesting and observations assimilation, and compare their performances. Some conclusions are given in Sect. 5. 


\section{Model set-up}

\subsection{Study area}

Our study area is the Gulf of Lions (GoL) (bathymetry shown in Fig. 1a), a large continental margin in the Northwestern Mediterranean Sea, where many small scale processes such as gyres and meanders along the canyon take place. The first internal Rossby radius is 7 to $11 \mathrm{~km}$ (Grilli and Pinardi, 1998), with local minima as small as a few $\mathrm{km}$. The GoL is influenced by different intense forcings. Two strong, cold and dry continental winds, the northern Mistral and northwestern Tramontane, are known to be key factors for the condition of the sea, leading to sea-surface cooling and the socalled Dense-Water Formation (see e.g. Lacombe and Tchernia, 1974; Millot, 1990, or more recently Estournel et al., 2003). Several rivers end in the GoL; the Rhône river is the most important one, and accounts for 80 to over $90 \%$ of the total discharge. The southern and eastern limits of the domain are open sea boundaries, with a major current flowing through: the Liguro-Provenal-Catalan (LPC) current, also called Northern Current. This current forms north of Corsica where the Eastern Corsican Current and Western Corsican Current join, and moves westward along the French coast. The LPC is formed of Modified Atlantic Water (down to $300-400 \mathrm{~m}$ ), and Levantine Intermediate Water (from 500 to $800 \mathrm{~m}$ ). It can be seen as the northern branch of the general cyclonic gyre of the north-western Mediterranean Sea. In order to conserve potential vorticity, the large-scale flow is constrained along the GoL shelf break, but instabilities can make it penetrate over the coastal region, partly controlling the shelf circulation. The LPC is largely responsible for the exchanges between the open sea and the shelf. This has been studied in the literature, e.g. Millot (1990); Estournel et al. (2003); Petrenko (2003) and review paper Millot (1999). In the winter, the LPC has been shown to be baroclinically unstable, with rapid variations in its position: the periods are of a few days to 20 days (Crépon et al., 1982). All these processes imply that a correct modeling of the GoL requires to take into account all scales, from small to large, in a full 3-D model.

\subsection{Hydrodynamic model}

Our study uses the GHER hydrodynamic model. It is a hydrostatic free-surface primitive equation model solving the prognostic variables of temperature, salinity, see surface elevation, horizontal velocity and turbulent kinetic energy, using the $\beta$-plane and Boussinesq approximations. Horizontally, it uses an Arakawa-C grid. In the vertical, it uses a double sigma coordinate, the limit between the two zones being at $170 \mathrm{~m}$ depth. Its integration scheme is conservative for tracers. Furthermore, the model uses mode splitting: for computational efficiency, the barotropic timestep is much smaller than the baroclinic one. The vertical turbulence uses a $\mathrm{k}$ turbulent kinetic energy closure scheme described in Nihoul et al. (1989). Further information about the GHER model can be found in Beckers (1991).

\subsection{Passive and interactive nesting}

As explained before, the Gulf of Lions is the siege of relatively small-scale processes that cannot be resolved by coarse resolution grids, but yet are (also) dependent on the large scales. For the simulations described below, a resolution of $1 / 100^{\circ}$ (approximately $1 \mathrm{~km}$ ) was desired. To resolve the open boundary problem, a system of nested grids was implemented. In order to avoid a high factor between the resolutions of the coarse and fine grids, an intermediate grid was also implemented, yielding two successive refinement factors of 5. The coarse resolution model covers the whole Mediterranean Sea with a resolution of $1 / 4^{\circ}$. The intermediate grid covers the area of the North-Western Mediterranean Sea, its eastern boundary being the Corsica and Sardinia islands, with a resolution of $1 / 20^{\circ}$. Finally, the third grid covers the area of the GoL with a resolution of $1 / 100^{\circ}$. The three grids are shown in Fig. 1.

The nesting procedure involves data exchanges between successive grids. In the so-called one-way nesting, the coarse grid model is interpolated on the fine grid to provide boundary conditions. The coarse grid does not use any information from the fine grid, thus, it can be run standalone first, the fine grid model being run afterward. This is useful for operational systems where the nested systems use different models, or are not run at the same place. This kind of implementation can be found in e.g. Pinardi et al. (2003), Korres and Lascaratos (2003), Echevin et al. (2003) or Zavatarelli and Pinardi (2003) for the Mediterranean Forecasting System pilot project (MFSPP). However, one-way nesting leads to the following disadvantage. If the simulation is run for a long period, discrepancies can appear between solutions of the grids, making the application of boundary conditions delicate, and possibly leading to instabilities in the fine grid model. If the fine grid model is reinitialized regularly (e.g. the Eastern Mediterranean basin subgrid is initialized every week in the MFS system (N. Skliris, private communication)), its small-scale features are lost. So-called variational initialization methods try to overcome this difficulty and are now used operationally, see e.g. Auclair et al. (2000, 2001). Another possibility is to reinitialize the fine model a few days before the actual forecast, so as to let the small-scale features develop (e.g. the North-Western basin subgrid in the MFS system performs an 8-day hindcast each week (C. Estournel and M. Lux, private communication)).

If both the coarse and fine models are run together, the fine model results can be averaged over each coarse grid cell in the overlapping area, where they replace the coarse model outputs. This yields the two-way or interactive nesting. Although the dynamic is different in the nested grids, inconsistencies are very less likely to appear between them. It has 
(b)
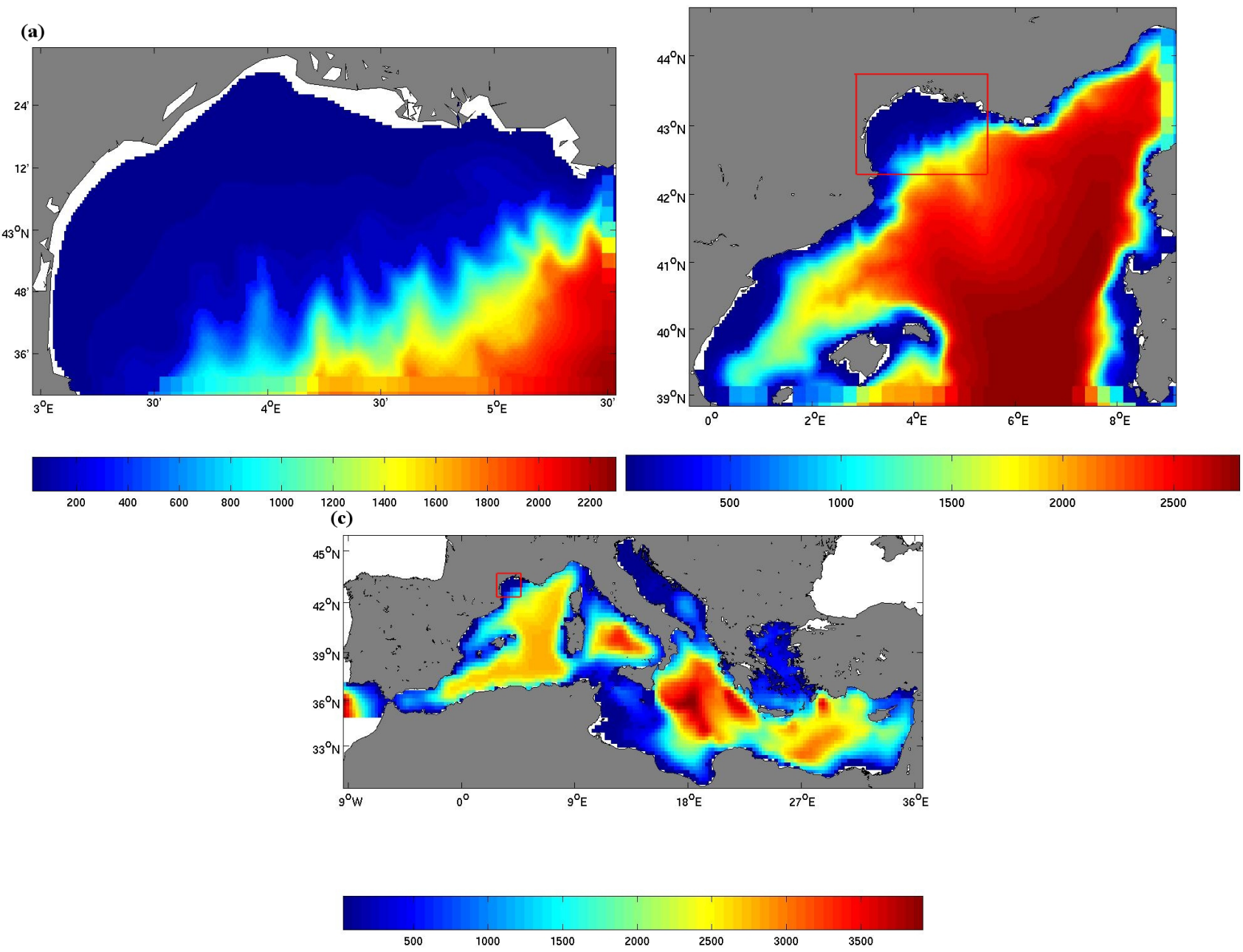

Fig. 1. Bathymetry of the 3 successive grids, in meters (a) Gulf of Lions (GoL), (b) North-Western Mediterranea (intermediate grid), (c) Mediterranean Sea (coarse grid). The correspond model grid resolution are respectively $0.01^{\circ}, 0.05^{\circ}$ and $0.25^{\circ}$ (for both horizontal directions). Red boxes in a grid indicate the position of the GoL grid.

been shown that two-way nesting yields more realistic representations of the mesoscale features inside the fine grid domain, and also has positive effects outside the domain (Barth et al., 2005).

Let's note that incoherences can also appear due to the fact that lateral open-sea boundary condition for the high resolution model are obtained by interpolation of the coarse resolution model outputs, as shown in Auclair et al. (2001). This problem was not further examined here.

In the present implementation, no refinement is used in the vertical. At the boundary of successive domains, the bathymetry is kept constant over the fine grid cells, corresponding to one coarse cell (as can be seen in Fig. 1). Over this boundary band, the land-sea mask is also identical for both grids. The original bathymetry is also smoothed more in the coarse grid than in the GoL grid. All the details about the nesting procedure can be found in Barth et al. (2005).

\subsection{Model implementation and results}

Further details of the implementation used for our study are given below.

The bathymetry is the Smith and Sandwell (1997) bathymetry. A model covering the whole Mediterranean Sea is started from MODB climatological initial conditions, and spun up for 10 years. Interpolation and averaging then yield the initial conditions of the 3 model grids described above. The same timestep is used in all 3 grids: $3 \mathrm{~s}$ (barotropic mode) and $3 \mathrm{~min}$ (baroclinic mode). We use a relaxation term towards the MODB/MEDAR4 climatology. Starting on 1 January 1998, the 3 models are spun up one month using two-way nesting. We use climatological Rhône river discharges (Tusseau and Mouchel, 1994). Interactive surface fluxes are computed using bulk formula; atmospherical data are the 6-hourly European Centre for Medium-Range 

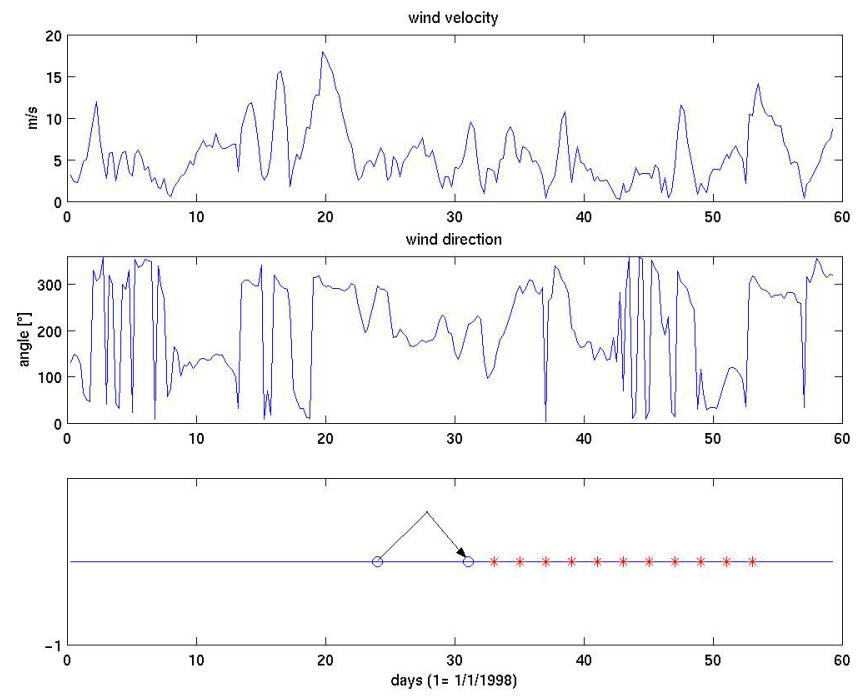

Fig. 2. (a) Average ECMWF-reanalysis wind direction over the Gulf of Lions (b) wind velocity (c) data assimilation cycles on the same time-scale. The blue circles represent the false and correct initial condition used to start the twin experiment on 30 January, while red stars represent assimilation cycles.

Weather Forecasts (ECMWF) reanalysis fields with a spatial resolution of a half degree. The wind speed and direction, averaged over the GoL grid, is shown in Figs. 2a and b. It can be seen that $3 \mathrm{Mistral} /$ Tramontane wind events take place around 14, 16-17 and 20 January. Other, similar or smaller events take place in February. It should be noted however that these average wind values do not correspond to a wind field homogeneous over the GoL. Finally, on 30 January, the twin experiment runs begin.

Although the purpose of this study is not to examine the hydrodynamics of the GoL itself, but rather to examine data assimilation in nested grids, we still present a brief summary. The results in the intermediate-resolution grid (Fig. 3a) clearly show the cyclonic gyre of the Western Mediterranean Sea. Its northern part, the LPC shows a width of 30 to $40 \mathrm{~km}$, in good agreement with the literature. Its current velocities are about $30 \mathrm{~cm} \mathrm{~s}^{-1}$ and the associated transport is about $1.3 \mathrm{~Sv}$, which is slightly less than the maximum values usually found for the winter in the literature, of 1.5 to $2 \mathrm{~Sv}$ (see e.g. Millot, 1999). The LPC is of course also visible in the lower part of the high-resolution grid (Fig. 3b), as well as the strong meanders detaching from it at various places. Figure 4 shows the salinity along the $\mathrm{A}^{\prime}-\mathrm{A}$ line in Fig. $3 \mathrm{~b}$, just next the Rhône river mouth. The salinity in the GoL is strongly influenced by the river plume, with values as low as 35.5 psu just at the river mouth. Along the shelf break, a vein of Levantine Intermediate Water (LIW) shows good agreement with the literature (e.g. Sparnocchia et al., 1995; Millot, 1999). It has a high salinity (38.45 psu in our simulation, compared to
$38.6 \mathrm{psu}$ in the literature), and its depth ranges from 300 to $700 \mathrm{~m}$, when it can go from 200 up to $1000 \mathrm{~m}$ in the literature. Below the LIW, the Western Mediterranean Deep Water (WMDW) has a lower salinity (38.3 psu compared to 38.4 in the literature). The temperature field shows the same water masses, also in good agreement with the literature. Finally, let's note that further away from the Rhône river mouth, the GoL waters are well mixed (not shown).

\subsection{Assimilation scheme}

We will use a Reduced-Rank Square Root (RRSQRT) assimilation scheme, described in Verlaan and Heemink (1997). It can be shown that the analysis of this filter is mathematically equivalent to the Singular Evolutive Extended Kalman (SEEK) filter, described e.g. in Pham et al. (1998); Brasseur et al. (1999). Only the first- and second-order moments of the error statistics are retained. Using the RRSQRT filter implies that we suppose that the processes can be considered quasilinear, and the model error is approximately gaussian. This is obviously not the case, as shown in Auclair et al. (2003). However, for relatively short time forecasts, we still will use this widespread approach.

If the model state vector dimension is $n$, instead for the model error covariance matrix $\mathbf{P}$ to have a rank $n \times n$, we can suppose it has only a rank $n \times r$, with $r \ll n$; hence it can be written as $\mathbf{P}=\mathbf{S S}^{T}$, where $\mathbf{S}$ is a $n \times r$ matrix. As a widely used approximation introduced in Pham et al. (1998), we will take the model variability in time as an estimation for its error. Then, the columns of $\mathbf{S}$ can be written as the first $r$ principal components, also called empirical orthogonal functions (EOFs); here, we take $r=20$ as it is reasonable trade-off, allowing to represent the errors fairly well while keeping computational cost low enough. As explained in Sect. 2.1, the processes involved in the GoL cannot be described otherwise than fully 3-D. Therefore, 3-D EOFs are calculated from the daily model states, from 2 model runs, one covering January and February 1997 and one covering January 1998. They are computed at the same time over 3 variables (T, S and $\eta$ ) and over the 3 grids, after the temporal mean has been removed from all the fields. In the state vector and EOFs, each point uses a norm equal to the product of the corresponding grid cell volume and the variables variance. Some parts of the first EOF are shown in Fig. 5.

It has been shown recently (Auclair et al., 2003) that a better data assimilation scheme, or at least a better errorspace, would be obtained by EOFs built from ensemble runs obtained by perturbing variables of the simulation, such as the atmospheric forcings, the forcing field along the open boundary, the initial state (and in particular the position and intensity of the LPC), model parameters and the bathymetry.

It should also be noted that the limited amount of "directions" that we use to build the errorspace, will probably not be able to correct all the errors encountered. In particular, 


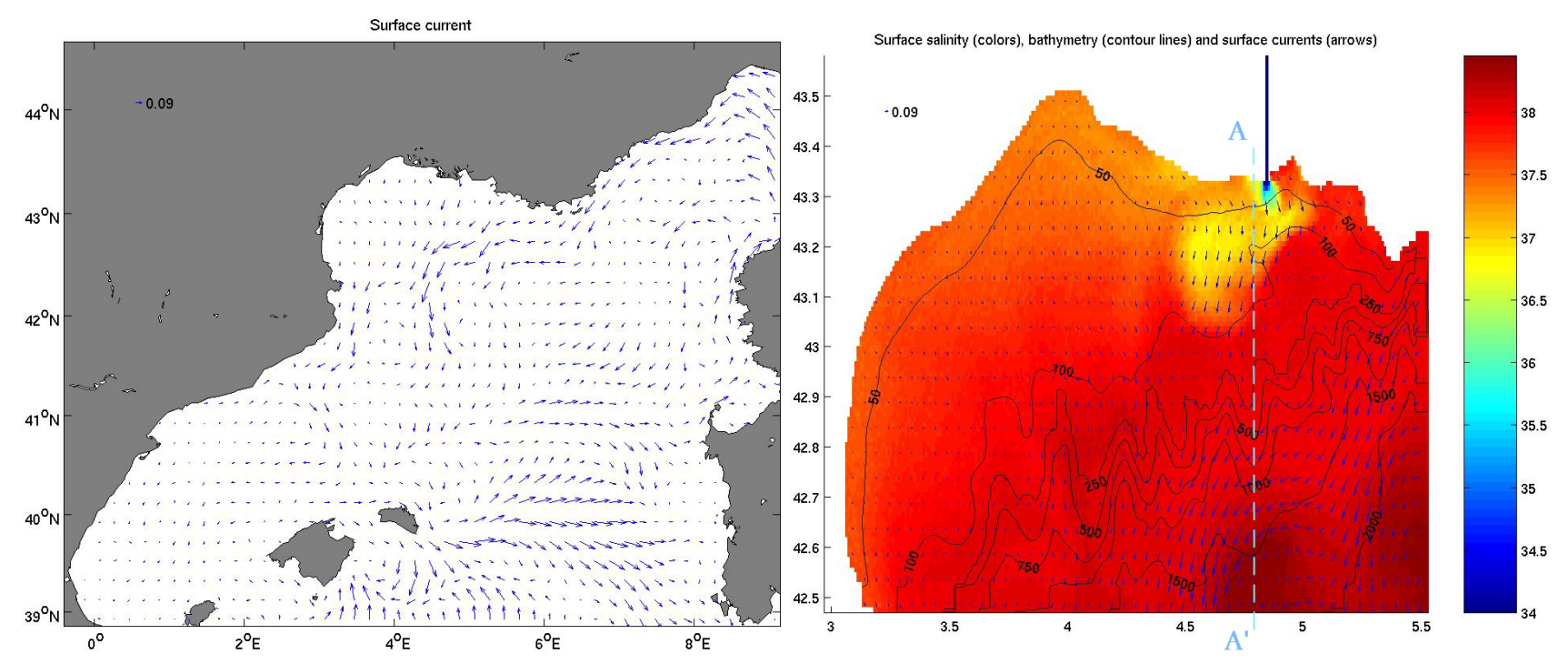

Fig. 3. Example of model results: plots from 17 January 1998. (a) Surface current velocity $\left[\mathrm{m} \mathrm{s}^{-1}\right]$ in the intermediate grid. The cyclonic gyre and LPC current are clearly visible. (b) The arrows represent surface currents $\left[\mathrm{m} \mathrm{s}^{-1}\right]$, the color represent surface elevation [m] and the contour lines represent isobaths [m]. The LPC follows the shelf break. Following Tramontane/Mistral wind bursts on 14 and 16 January, an intense current moves surface waters (and the Rhône plume in particular) away from the coastline.

the high variability in the Rhône river plume will probably not be resolved by our errorspace basis.

A final approximation will be not to update $\mathbf{P}$ during the simulation. Because of the procedure followed to obtain it, $\mathbf{P}$ is representative for the period covering January and February. However, in the complete RRSQRT or SEEK filters, the error covariance is updated by the model (generally increasing it) and by the assimilation scheme (decreasing it). Thus, the ratio of the eigenvalues of the projection of $\mathbf{P}$ in the observation space, and the eigenvalues of $\mathbf{R}$ (the observations error covariance matrix), is modified during the simulation; this is not the case in our experiment as both $\mathbf{P}$ and $\mathbf{R}$ are constant in time.

Our model state vector contains temperature $(\mathrm{T})$ and salinity (S) at each 3-D gridpoint, as well as sea surface elevation $(\eta)$. Thus, whenever data is assimilated, this leads to corrections on the T, S, and $\eta$ variables. These corrections are multiplied by a radial gaussian function centered on the corresponding observation in order to limit the spatial extent of the correction that an observation can yield. In the present case, the gaussians extent $\sigma$ is put to $100 \mathrm{~km}$. Let's notice that if $\mathbf{P}$ would accurately represent the model error covariance, this step would not be necessary; unphysical long-range correlations would not be present in the computed statistics. Finally, the geostrophic velocity correction (corresponding to the T, $\mathrm{S}$ and $\eta$ corrections) is computed and applied to the model horizontal velocity variables $U$ and $V$.

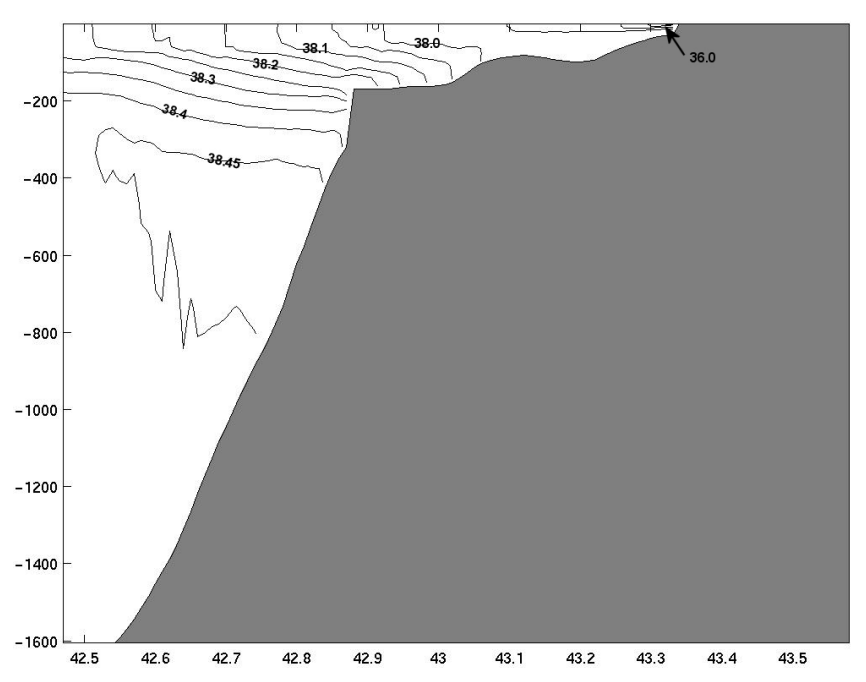

Fig. 4. Salinity along the $A^{\prime}-A$ line in Fig. 3b. The salinity in the GoL is influenced by the Rhône. A LIW vein is clearly visible along the shelf break.

\section{Twin experiment}

In the present section, a twin experiment is set up. The model run shown in the previous section is used as the reference run. Another run will start from different initial conditions, being the ocean state from January, 23 instead of 30 January 1998 (see Fig. 2c). This choice ensures that the initial conditions are physically balanced. The grids are also coherent with each other, since the reference run uses interactive nesting. 
(a)

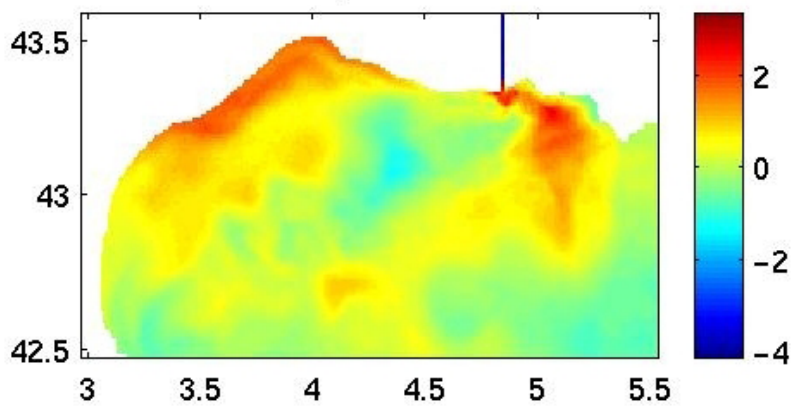

(c) Salinity

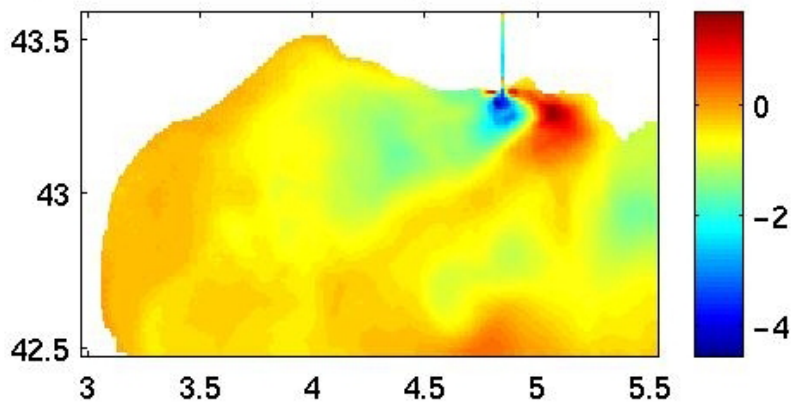

(e) Surface Elevation

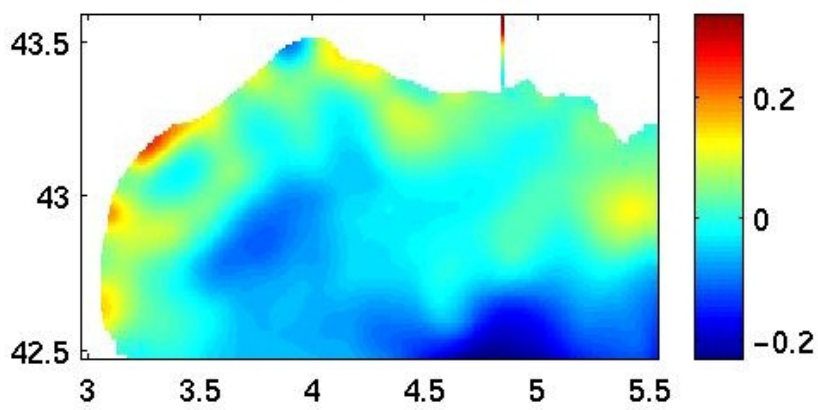

(b) Temperature

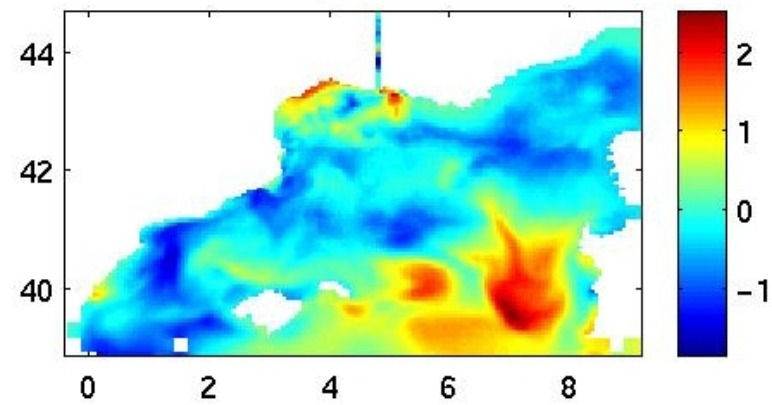

(d) Salinity

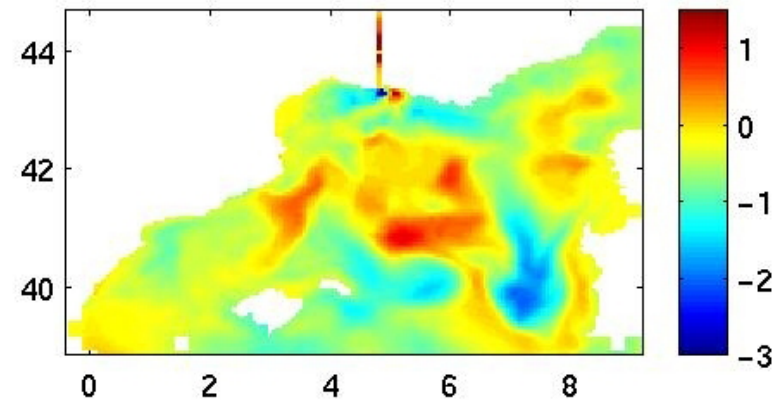

(f) Surface Elevation

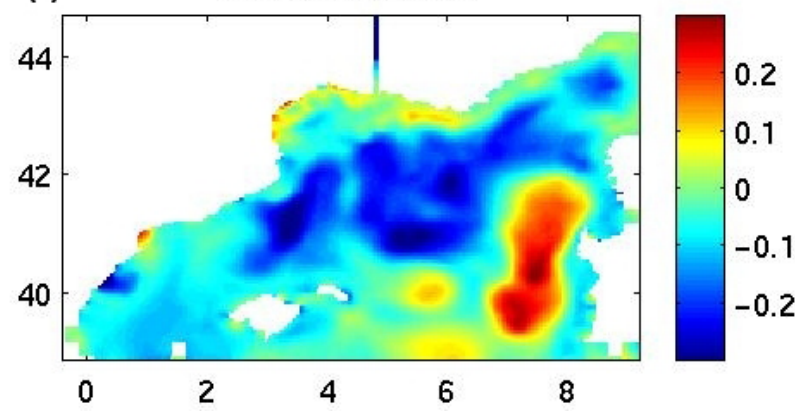

Fig. 5. Surface plot of the temperature in ${ }^{\circ} \mathrm{C}(\mathbf{a}, \mathbf{b})$, surface salinity in psu (c, d) and elevation in $\mathrm{m}(\mathbf{e}, \mathbf{f})$ parts, in the GoL (a, c, e) and in the Intermediate grid $(b, d, f)$, of the first multigrid multivariate 3-D EOF, calculated after removing the temporal mean. The 1st EOF shows relatively large-scale structures, when EOFs of higher order represent structures with a smaller scale (not shown).

Some atmospheric forcings (wind velocity, air temperature and cloud coverage) are also modified in the following way. The real fields are decomposed as weighted sums of EOFs, which are obtained from a year time-series. It appears that 30 EOFs are needed to accurately describe the cloud coverage field (95\% of variance), 10 to 20 for air temperature and about 80 for wind velocity (see Fig. 6). For all 4 fields however, we used 100 principal components, the computational cost being low. The modified fields are then obtained by multiplying the real weights by a random factor included in $[1-\epsilon, 1+\epsilon]$. This random factor is kept constant at all times in order to avoid large, unphysical variations in the forcing fields. Adequate fields were obtained by using $\epsilon$ equal to $0.4,0.5$ and 0.35 , respectively for the wind velocity, cloud coverage and air temperature fields.
Every 2 days starting on January 31st, pseudoobservations are assimilated in a perturbed run in order to bring it as close as possible to "reality" (assimilation cycles are represented by red stars in Fig. 2c). As pseudo-data, we assimilate Sea Surface Temperature (SST) over the whole GoL, and Sea Surface Height (SSH) over a pseudo-track in the Gulf of Lions. As an example, the observations to use during the first assimilation cycle (on 31 January) are shown in Fig. 7. The error covariance corresponding to these observations is represented by a diagonal matrix $\mathbf{R}$, with values equal to the square of $1^{\circ} \mathrm{C}$ (for temperature) and $0.4 \mathrm{~m}$ (for surface elevation). 


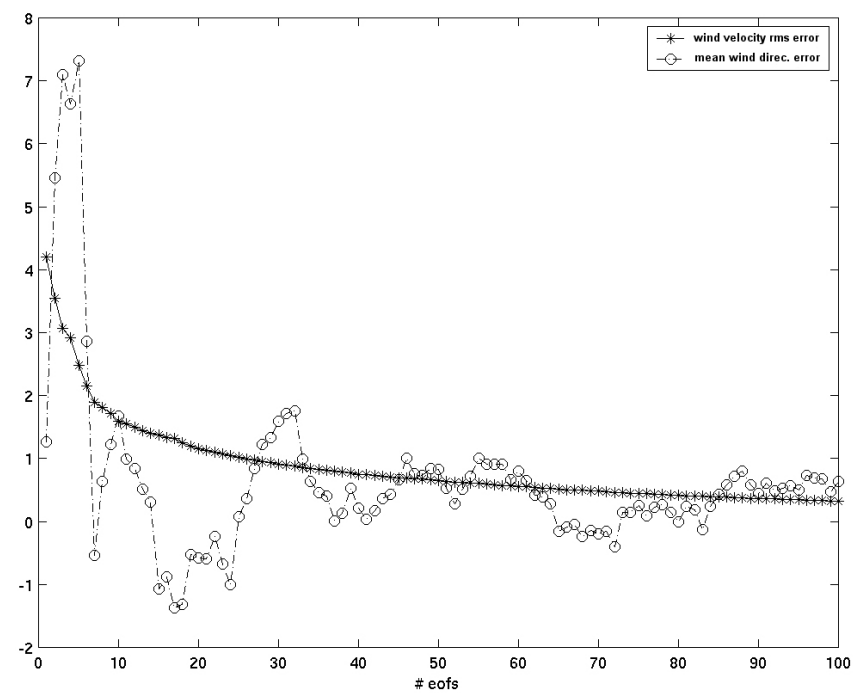

Fig. 6. Error between the actual wind field, and its recomposition using the $\mathrm{N}$ first EOFs, as a function of $\mathrm{N}$ (a) rms error of the velocity $[\mathrm{m} / \mathrm{s}]$ (b) mean direction error $\left[{ }^{\circ}\right]$ (means are calculated spatially over the whole Mediterranean grid).

\section{Comparison of different setups}

If observations are available in the area covered by the fine grid (the GoL), they could be assimilated in the coarse (and intermediate) grid, or in the fine grid, or both. Our purpose is to examine which setup is the most efficient in the context of an operational system where only one-way nesting is currently used. Therefore, we define the following 4 study cases:

- case 1: the nesting is two-way, and data is assimilated in the fine grid. The corrections are automatically fed back to the other grids via the two-way nesting. As mentioned before, two-way has many advantages in- and outside the coastal domain; but it is not feasible in most operational configurations such as the one used in the MFSTEP project.

- case 2 will use one-way nesting, observations are still assimilated in the fine grid only.

- case 3 also uses one-way nesting, but data is assimilated in all the grids at the same time.

- in case 4, one-way nesting still is used; data is assimilated in the coarse grid only. Let's note that since the observations assimilated in the coarse grid are still physically located in the Gulf of Lions, information should be transported to the intermediate-resolution model through the boundary conditions, and hereafter transported to the high-resolution model.
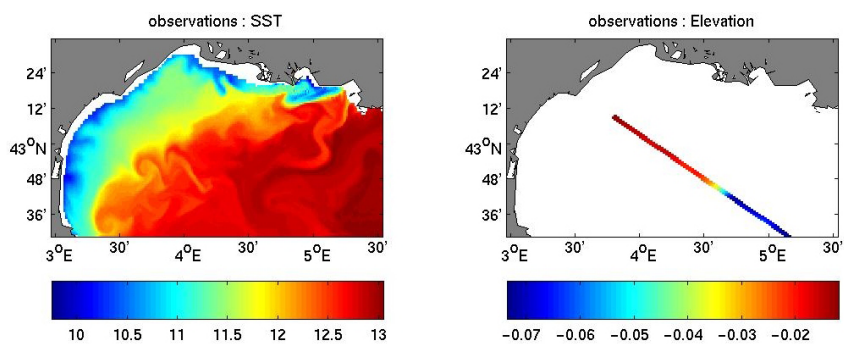

Fig. 7. Pseudo-observations coming from the reference run: (a) Sea Surface Temperature $\left[{ }^{\circ} \mathrm{C}\right]$, (b) Sea Elevation corresponding to a typical satellite track $[\mathrm{m}]$

- Moreover, we will define a fifth case, based on the work of Barth et al. (2006). In this case, all 3 state vectors from the 3 grids are assembled in a unique state vector. Since the EOFs have been calculated over the 3 grids together too, perfect correlation is assured between data, located at the same physical points in different grids. Hence, an observation automatically yields coherent corrections in the 3 grids. If an evolutive assimilation scheme were to be used (i.e. the model would be used to update $\mathbf{P}$ in time), the model error covariance update equations would also yield "errorspace" feedback.

Let's note that in cases 2, 3 and 4, one-way nesting is used, so that discrepancies could appear between grids, ultimately leading to instabilities in the coastal model. Other instabilities may appear, as mentioned before, in the Rhône river plume after each assimilation cycle. Therefore, the following defensive procedure was implemented. Before and after assimilation, the Brunt-Vasala frequency $N^{2}$ is calculated for each gridpoint. The spatial mean of its square values is also computed, separately for positive (stable) and negative (unstable) values of $N^{2}$. For a point where $N^{2}$ is positive, if $N^{2}$ after the assimilation is smaller than 3 times the mean square value of all positive values in the grid, the assimilation correction is completely applied; if $N^{2}$ is larger than 10 times the mean square value, the correction is not applied. For values of $N^{2}$ between 3 and 10 times the mean square value, the correction decreases linearly. The same procedure is applied for points with a negative $N^{2}$ frequency. In our study, this yields masks where the corrections in the Rhône plume are almost systematically put to zero, confirming our suspicion that the errorbase would not be able to describe the plume variability (Fig. 8).

As an example, the correction to the surface temperature in the GoL, after the first assimilation cycle in case 5, is shown in Fig. 9. The corresponding assimilated data are shown in Fig. 7.

Figure 10 shows plots of the rms error between the reference run, and the runs corresponding to cases 1 to 5 as well as 

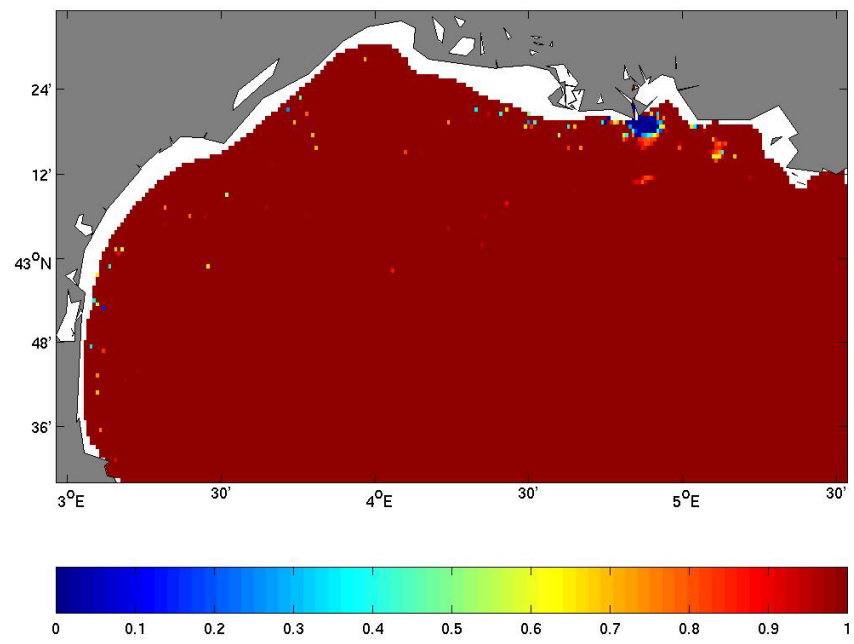

Fig. 8. An example of a mask used to multiply the correction, and computed from the $\mathrm{N} 2$ field after assimilation. The shown mask is calculated for the first assimilation cycle.

a free run (without data assimilation). The averages are calculated over the grid points where observations are available. All the runs start from the same rms error, which is the rms between the "right" and alternative initial conditions. It can be seen that case 1 (the simulation with interactive nesting) presents rms errors very close to case 5 (the simulation with feedback, but where corrections are automatically copied to the 3 grids, since all 3 grids are comprised in the state vector). However in our experiment, we kept the model error covariance matrix $\mathbf{P}$ constant. If it would be modified by the assimilation procedure, those modifications would still be coherent, in case 5, for covariances between identical physical points located in different grids (in opposition to what would happen in the other 4 cases). Hence, the assimilation of observations in subsequent assimilation cycles would probably be more accurate. Cases 1 and 5 both show much smaller errors than the 3 cases using one-way nesting. This indicates that the errors, which were not corrected in the global and intermediate model, are advected back to the local model through the boundary conditions. It can also be seen that the differences between cases 2 and 3 (assimilate in the GoL only, or in all 3 grids), are very small; indicating that the corrections made in the (intermediate and) global model are not sufficient to improve the boundary conditions significantly. In any way, their effect on the rms errors is much smaller than the assimilation of data in the local grid. Small corrections in the coarse grid are due to the fact that these fields are already close to the observations, with respect to the error covariances. Unless the correction in the coarse grids becomes much more important (i.e. the fields in the coarse grid part further away from "reality"), it is thus not very useful to assimilate observations in the coarse grid. Finally, case 4 shows the largest rms errors of all. Corrections are only brought to the coarse

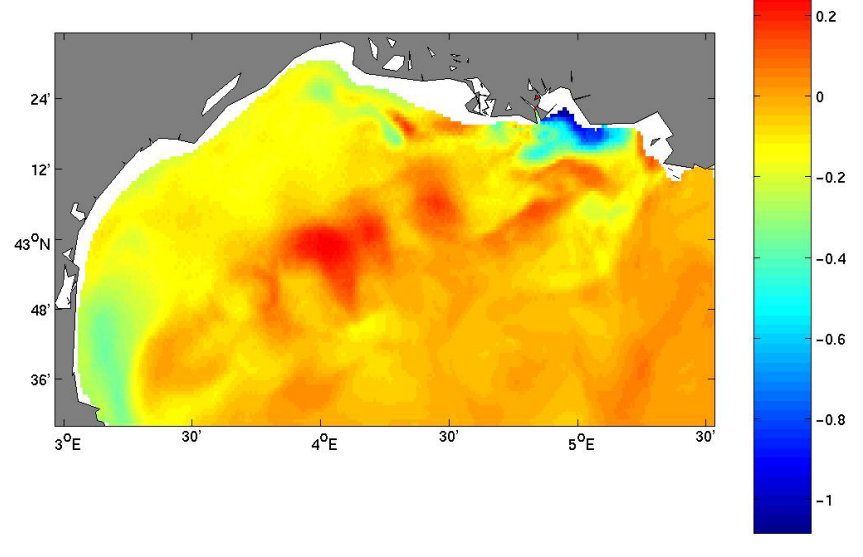

Fig. 9. Correction yielded by the first assimilation cycle on the Sea Surface Temperature $\left[{ }^{\circ} \mathrm{C}\right]$

grid; they need to propagate via the intermediate model, to the local model. There is no immediate change in the rms errors of the local model at assimilation times. Since the rms error of in case 4 is ultimately smaller than the one in the free run, it seems the information (assimilated in the global model) slowly arrive in the local model anyway.

It can also be noted that the error on surface elevation is small (a few centimeters) at all times, even in the "free" run. During assimilation cycles, it is reduced by a factor close to 2 , but in between each assimilation cycle, the model causes new errors of the same order as the correction, leading to an oscillating rms error curve. However, the maximum error remains of the order of $3 \mathrm{~cm}$.

It is interesting to examine the rms errors between the reference run and the other cases, with the averages calculated over other location than those where observations are available (of course, this is only possible in the framework of a twin experiment). In particular, we show the rms curve calculated over the whole 3-D grid in Fig. 11. As example, we used case 5. As can be seen, the data assimilation cycles reduce the overal rms error, even in points where no data was assimilated. In fact, the procedure also reduces rms errors on the salinity variable, although no salinity observations are available. This shows that the errorspace correcly represents the statistical covariance between different model variables. Only during the fifth assimilation cycle (on 8 February 1998, at midnight), the correction on SST and surface elevation along a satellite track, statistically yields a salinity correction which actually increases the salinity rms error. It can be seen that the surface elevation mismatch is, for that assimilation cycle, very important (almost 3 times larger than during the first assimilation cycle). 

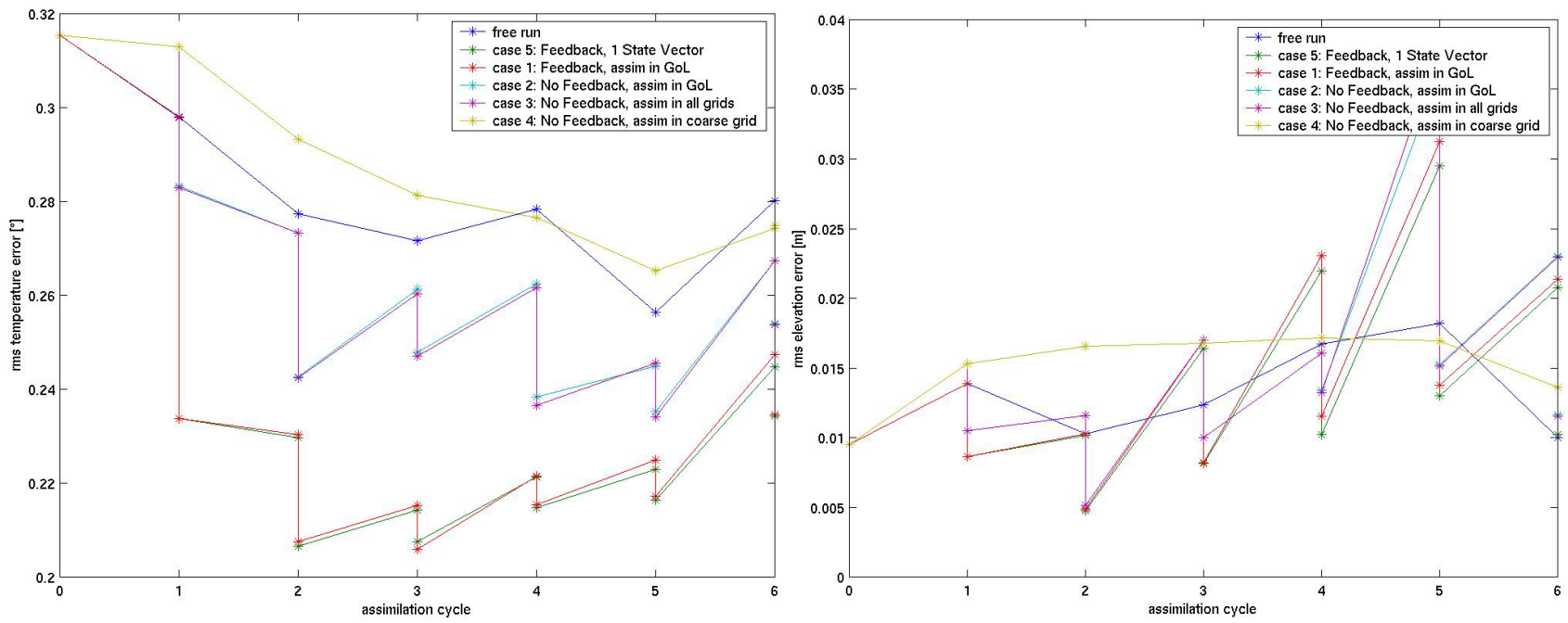

Fig. 10. Evolution of the rms error in time, between the reference run and the perturbed runs, the latter being the free run (blue curve), case 1 (red curve), case 2 (turquoise curve), case 3 (purple curve), case 4 (yellow curve), and case 5 (green curve), showing (a) SST (b) Surface elevation. The stars represent assimilation cycles.

\section{Conclusions}

We have studied the impact of data assimilation in a nested hydrodynamic model, assessing the question whether the available observations should be assimilated in the highresolution, local grid, or in the coarse-resolution grid. Using twin-experiments with different test-cases, we obtained some general conclusions, e.g. that

- two-way nesting moves the model towards "reality" faster than one-way nesting. Indeed, when new information is added in any grid, it is transmitted to all the other grids; in the one-way nesting paradigm, information only goes from low-resolution to high-resolution grids.

- the RRSQRT filter that we used, yields satisfactory results, even though we chose a low errorspace dimension (20). Only in places with very high variability (such as a river plume or some points along the coastlines), the filter could not capture the model variability and hence, we artificially diminished the correction in those points, because they were expected to be incorrect.

- using multi-variable state-vectors, we have corrected all variables by observing only some of them. Using a full 3-D matrix, we have also corrected variables over the whole grid, although only surface variables were available.

Supposing that the global model is approximately correct (i.e. it feeds the local model with boundary conditions "not too far" from reality), we showed that the high-resolution

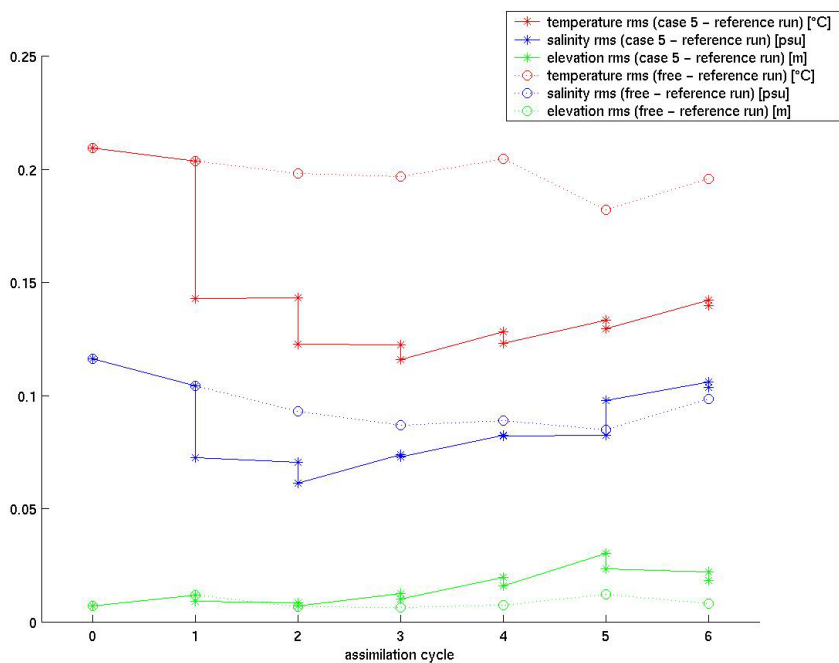

Fig. 11. Evolution of the rms error in time, between the reference run and case 5 , of the rms error calculated over the entire 3-D field of temperature (blue curve), the entire 3-D field of salinity (green curve), the whole surface elevation field (red curve). The stars represent assimilation cycles.

model is better corrected when available observations are assimilated immediately in that grid, rather than to assimilate them in the coarse-resolution grid and transport the information in the local model via boundary conditions. And if the data is assimilated in the high-resolution model, it is then of little use to also have assimilated it in the global model (this is even less useful when using interactive nesting). 
Acknowledgements. This work was performed in the frame of the MFSTEP project (see http://www.bo.ingv.it/mfstep). This is MARE publication MARE097.

Edited by: N. Pinardi

\section{References}

Auclair, F., Casitas, S., and Marsaleix, P.: Application of an inverse method to coastal modeling, J. Atmos Oceanic Technol., 17, 1368-1391, 2000.

Auclair, F., Marsaleix, P., and Estournel, C.: The penetration of the Northern Current over the Gulf of Lions (Mediterranean) as a downscaling problem, Oceanologica Acta, 24, 529-544, 2001.

Auclair, F., Marsaleix, P., and De Mey, P.: Space-time structure and dynamics of the forecast error in a coastal circulation model of the Gulf of Lions, Dynamics of Atmospheres and Oceans, 36, 309-346, 2003.

Barth, A., Alvera-Azcárate, A., Beckers, J.-M., Rixen, M., and Vandenbulcke, L.: Multigrid state vector for data assimilation in a two-way nested model of the Ligurian Sea, J. Marine Syst., in press, 2006

Barth, A., Alvera-Azcárate, A., Rixen, M., and Beckers, J.-M.: Two-way nested model of mesoscale circulation features in the Ligurian Sea, Progress In Oceanography, 66, 171-189, 2005.

Beckers, J.-M.: Application of a 3D model to the Western Mediterranean, J. Mar. Syst., 1, 315-332, 1991.

Blayo, E. and Debreu, L.: Adaptive mesh refinement for finite difference ocean model: some first experiments, J. Phys. Ocean., 29, 1239-1250, 1999.

Brasseur, P., Ballabrera, J., and Verron, J.: Assimilation of altimetric data in the mid-latitude oceans using the Singular Evolutive Extended Kalman filter with an eddy-resolving, primitive equation model, J. Mar. Syst., 22, 269-294, 1999.

Crépon, M., Wald, L., and Monget, J.: Low-frequency waves in the Ligurian Sea during December., J. Phys. Oceanogr., 87, 595600, 1982.

Echevin, V., Crépon, M., and Mortier, L.: Simulation and analysis of the mesoscale circulation in the northwestern Mediterranean Sea, Ann. Geophys., 21, 281-297, 2003, http://www.ann-geophys.net/21/281/2003/.

Estournel, C., Durrieu de Madron, X., Marseleix, P., Auclair, F., Julliand, C., and Vehil, R.: Observation and modeling of the winter coastal oceanic circulation in the Gulf of Lion under wind conditions influenced by the continental orography (FETCH experiment), J. Geophys. Res., 108, 8059-8076, 2003.

Fox, A. D. and Maskell, S. J.: Two-Way Interactive Nesting of Primitive Equation Ocean Models with Topography, J. Phys. Oceanogr., 25, 2977-2996, 1995.

Fox, A. D. and Maskell, S. J.: A nested primitive equation model of the Iceland-Faeroe front, J. Geophys. Res., 101, 18 259-18 278, 1996.

Ginis, I., Richardson, R. A., and Rothstein, L. M.: Design of a Multiply Nested Primitive Equation Ocean Model, Mon. Wea. Rev., 126, 1054-1079, 1998.
Grilli, F. and Pinardi, N.: The computation of Rossby radii of deformation for the Mediterranean sea, MTP News No. 6, March, 1998.

Korres, G. and Lascaratos, A.: A one-way nested eddy resolving model of the Aegean and Levantine basins: implementation and climatological runs, Ann. Geophys., 21, 205-220, 2003, http://www.ann-geophys.net/21/205/2003/.

Lacombe, H. and Tchernia, P.: Les zones de formation d'eau profonde océanique: Caractères-processus-problèmes, in: Processus de formation des eaux océaniques profondes, Colloq. Int Centre Natl. Rech. Sci., 215, 249-262, 1974.

Millot, C.: The Gulf of Lions' hydrodynamics, Cont. Shelf Res. $10,885-894,1990$

Millot, C.: Circulation in the Western Mediterranean Sea, J. Mar Syst., 20, 423-442, 1999.

Nihoul, J. C. J., Deleersnijder, E., and Djenidi, S.: Modelling the general circulation of shelf seas by 3D $k-\epsilon$ Models., Earth Sci. Rev., 26, 163-189, 1989.

Oey, L.-Y. and Chen, P.: A Nested-Grid Ocean Model: With Application to the Simulation of Meanders and Eddies in the Norwegian Coastal Current, J. Geophys. Res., 97, $20063-20$ 086, 1992.

Petrenko, A.: Circulation features in the Gulf of Lions, NW Mediterranean Sea; importance of inertial currents., Oceanologica Acta, 26, 323-338, 2003.

Pham, D. T., Verron, J., and Roubaud, M. C.: A singular evolutive extended Kalman filter for data assimilation in oceanography., J. Mar. Syst., 16, 323-340, 1998.

Pinardi, N., Allen, I., Demirov, E., De Mey, P., Lascaratos, A., Le Traon, P.-Y., Maillard, C., Manzella, G., and Tziavos, C.: The Mediterranean ocean forecasting system: first phase of implementation (1998-2001), Ann. Geophys., 21, 3-20, 2003, http://www.ann-geophys.net/21/3/2003/.

Smith, W. H. F. and Sandwell, D. T.: Global Sea Floor Topography from Satellite Altimetry and Ship Depth Soundings, Science, 277, 1956-1962, 1997.

Spall, M. A. and Robinson, A. R.: A new open-ocean hybrid coordinate primitive equation model, Math. Comput., 31, 241-269, 1989.

Spall, M. A. and Holland, W. R.: A nested primitive equation model of oceanic application, J. Phys. Oceanogr., 21, 205-220, 1991.

Sparnocchia, S., Picco, P., Manzella, G. M. R., Ribotti, A., Copello, S., and Brasey, P.: Intermediate water formation in the Ligurian Sea, Oceanologica Acta, 18, 151-162, 1995.

Tusseau, M. and Mouchel, J.: Nitrogen inputs to the Gulf of Lions via the Rhône river, in: Water Pollution Research Reports, Proceedings of the EROS 2000 Workshop, Hamburg, edited by: Martin, J. and Barth, H., commission of the European Communities, 1994

Verlaan, M. and Heemink, A. W.: Tidal flow forecasting using reduced rank square filters, Stochastic Hydrology and Hydraulics, 11, 349-368, 1997.

Zavatarelli, M. and Pinardi, N.: The Adriatic Sea modelling system: a nested approach, Ann. Geophys., 21, 345-364, 2003, http://www.ann-geophys.net/21/345/2003/. 